\title{
THE BLOOD SUPPLY OF MALIGNANT PULMONARY NEOPLASMS*
}

\author{
BY \\ L. CUDKOWICZ $\dagger$ AND J. B. ARMSTRONG \\ From the Department of Medicine, Postgraduate Medical Schsol, London
}

(RECEIVED FOR PUBLICATION JANUARY 11, 1952)

Wood and Miller (1938) obtained radiographic evidence post mortem of proliferation of the bronchial arteries in primary pulmonary neoplasms and they regarded this systemic vascularization as responsible for the frequency of haemoptyses associated with bronchial carcinomata. Metastatic lung tumours showed no such pattern, and they believed that this could be an explanation for the absence of haemoptyses in secondary lung tumours. Wright (1938) found the same profuse bronchial blood supply to primary bronchial tumours, but contended that the blood supply of metastases in the lungs was similar.

With the aid of a post-mortem injection technique, which has already been described (Cudkowicz and Armstrong, 1951), the bronchial arteries were studied both radiographically and histologically in five cases of pulmonary neoplasm.

\section{Material}

A summary of the cases studied is given in Table I.

TABLE I

\begin{tabular}{|c|c|c|c|}
\hline Case & Sex & Age & Post-mortem Diagnosis \\
\hline 1 & $\mathbf{M}$ & 61 & $\begin{array}{l}\text { Carcinoma of the right middle lobe bronchus } \\
\text { with widespread bony and cerebral metastases }\end{array}$ \\
\hline 2 & $\mathbf{F}$ & 57 & $\begin{array}{l}\text { Carcinoma of right posterior basic bronchus, } \\
\text { secondary tumour in right medial basic } \\
\text { segment. pericardial infiltration, and throm- } \\
\text { bosis of inferior vena cava }\end{array}$ \\
\hline 3 & $\mathbf{M}$ & 53 & $\begin{array}{l}\text { Carcinoma of apical division of right upper } \\
\text { lobe bronchus and multiple cerebral meta- } \\
\text { stases }\end{array}$ \\
\hline 4 & $\mathbf{M}$ & 62 & $\begin{array}{l}\text { Carcinoma of apical bronchus of left upper } \\
\text { lobe with multiple skeletal metastases }\end{array}$ \\
\hline 5 & $\mathbf{F}$ & 85 & $\begin{array}{l}\text { Carcinoma of stomach, secondary tumour in } \\
\text { right lower lobe, erosion of lower thoracic } \\
\text { and abdominal aorta by tumour. Liver and } \\
\text { lymph node metastases }\end{array}$ \\
\hline
\end{tabular}

* Some of the material in this paper was incorporated in a thesis by $L$. C. which was accepted by the University of London for the M.D. degree in December, 1950

+ In receipt of a grant from the Medical Research Council

\section{RESULTS}

Primary Bronchial Carcinomata.-This groupi consists of three cases.

Case 1.-This patient, aged 61 , was clinic-ally believed to have a carcinoma of the right uppere lobe bronchus, and in the radiograph a larged shadow was visible in the region of the right upper $\vec{c}$ lobe. The fingers and toes were clubbed. As necropsy a large secondary growth was noted in the posterior half of the right sixth rib, which hado broken through the parietal pleura. The lung itsels was not involved, but pressure by the tumour dis placed the right upper lobe antero-medially. mass approximately $4 \times 3 \mathrm{~cm}$. was palpable in the right middle lobe.

The radiographic appearances of the injected bronchial arteries are shown in Fig. 1. A norma pattern was seen in the left lung. A dense arterial. network extended from the right side of the descending aorta, and coursed to the right hilum There a number of lymph nodes received a profuse blood supply. The remaining irregularly spaceds arteries continued to form a mesh of vessels around the tumour mass in the right middle lobe. The tumour mass itself was outlined by extravasate of injection material within its substance. Anas? tomotic filling of the pulmonary arterioles distal to the tumour had taken place. The distribution of: the bronchial arteries to the right upper and lowes lobe was normal. A right bronchogram showed that the tumour arose from the medial bronchus of the right middle lobe near its inception. The tumour occluded its lumen and partially stenosed the lateral bronchus.

Histological examination showed secondar deposits in the pretracheal and subcarinal lymph nodes. The cells were of the " oat cell" varietyo Large arterioles, filled with injection medium, wer $\Phi^{\circ}$ visible in the stroma and capsule of the involved lymph nodes. Large bronchial arteries were visible in the wall of the affected bronchus proximal to the tumour. At the edge of the 


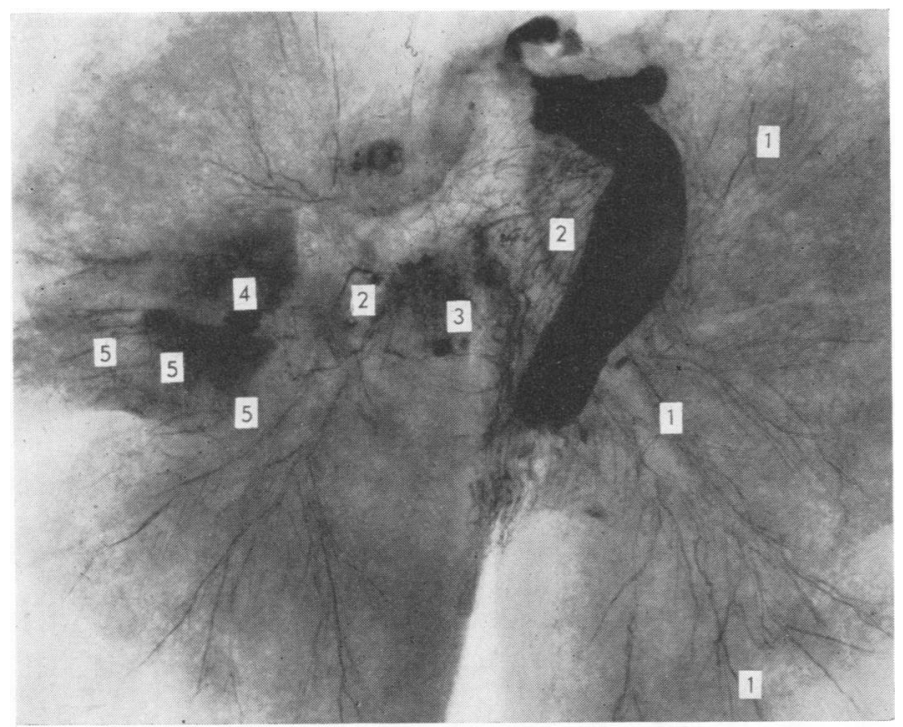

FIG. 1.-Radiograph of both lungs in Case 1 showing: 1, normal bronchial arterial pattern to the left lung; 2 , proliferation of the bronchial arteries to the right middle lobe with distortion of the normal pattern; 3, extravasation of the injection mass into lymph nodes at right hilum; 4, extravasation of injection mass into tumour; 5, anastomotic filing of peripheral pulmonary arteries. About $\frac{1}{3}$ of normal size

tumour these vessels branched out and continued along strands of collagenous tissue into the tumour substance. Within the tumour itself large vascular lacunae were visible, which were lined by a fine endothelium and filled with injection mass. Elsewhere considerable numbers of red blood corpuscles and extravasated bismuth granules from the injection mass were near large and dilated vessels (Fig. 2). In the adjacent lung tumour cells lay between the alveoli. The involved bronchus showed replacement of its wall by the tumour mass and crowding of the remaining epithelial remnants in its lumen. The accompanying pulmonary artery branch had extensive infiltration of its wall by tumour cells and occlusion of its lumen by thrombosis. The vasa vasorum in the adventitia were dilated and fully injected. They extended into the occluded pulmonary artery lumen. This recanalization of the pulmonary artery was more marked at the periphery of the middle lobe, and the pulmonary arterioles there were fully injected.

Case 3.-The clinical diagnosis of this male patient, aged 53, before his death was carcinoma of the right upper lobe bronchus with multiple cerebral metastases, which had led to personality changes and a left hemiparesis. He showed considerable clubbing of the fingers and toes. Necropsy confirmed the site of the tumour and the cerebral lesions. Radiography of the injected lungs showed good filling of the bronchial arteries which were normal in their distribution to the left lung. Pretracheal and subcarinal lymph nodes were outlined by the injection medium. A large number of bronchial vessels entered the right upper lobe. In the radiographs two white patches indicated tumour masses in the apical and posterior segments. The pattern of the bronchial arteries in the anterior segment and in the right middle and lower lobes was normal. At the periphery of the right upper lobe, distal to the tumour masses, anastomotic filling of the pulmonary arteries was evident (Fig. 3). Bronchography showed obstruction by the tumour of the apical and posterior bronchi of the right upper lobe. The histology disclosed a squamous-cell type of carcinoma in the right upper main bronchus just distal to the origin of the anterior superior bronchial division. The lumen of the bronchus was stenosed and tumour cells heavily infiltrated the wall and replaced the normal structures. Many large bronchial arteries were visible in the surrounding lung, and large bismuth-filled lacunae appeared to lie between aggregates of tumour tissue. The pulmonary artery accompanying the involved bronchus was thrombosed and its wall was heavily infiltrated with tumour cells. The lumen showed evidence of recanalization by proliferating and injected vasa vasorum, and the peripheral patent pulmonary artery branches were heavily injected.

Case 4.-This male patient, aged 62 , had a tumour in the left upper lobe bronchus and widespread skeletal metastases. At necropsy the left upper lobe was densely adherent to the parietal pleura and a tumour was palpable in the apical segment. Radiography of the injected left lung showed considerable enlargement of the bronchial arteries running towards the tumour and extravasation of the injection medium into its substance (Fig. 4). The histology showed very similar changes to those in the previous case. The pulmonary arteries in this case were not affected and no anastomotic filling was present.

Metastatic Tumours in THE Lung.-The last two cases can be thus grouped.

Case 2.-This female patient, aged 57, came to necropsy with the clinical diagnosis of carcinoma of the right lower lobe bronchus with involvement of the inferior vena cava leading to its obstruction. 


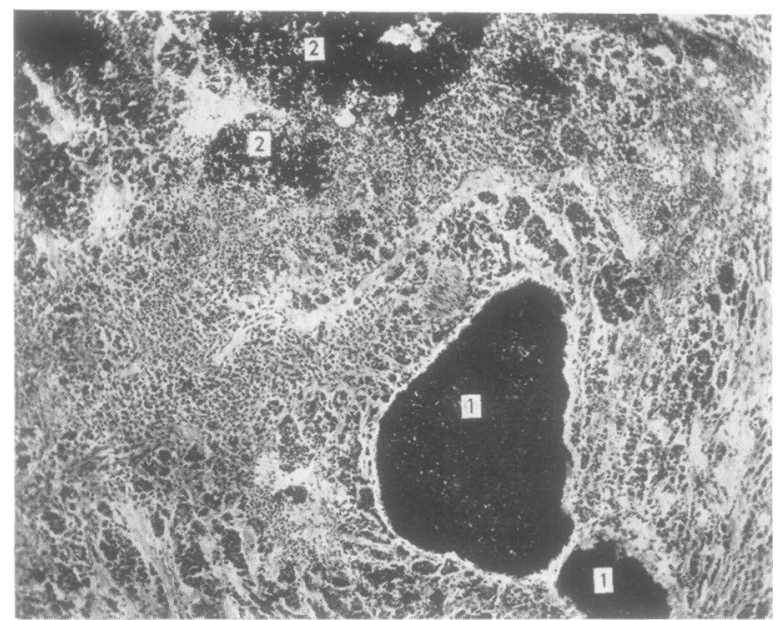

FIG. 2

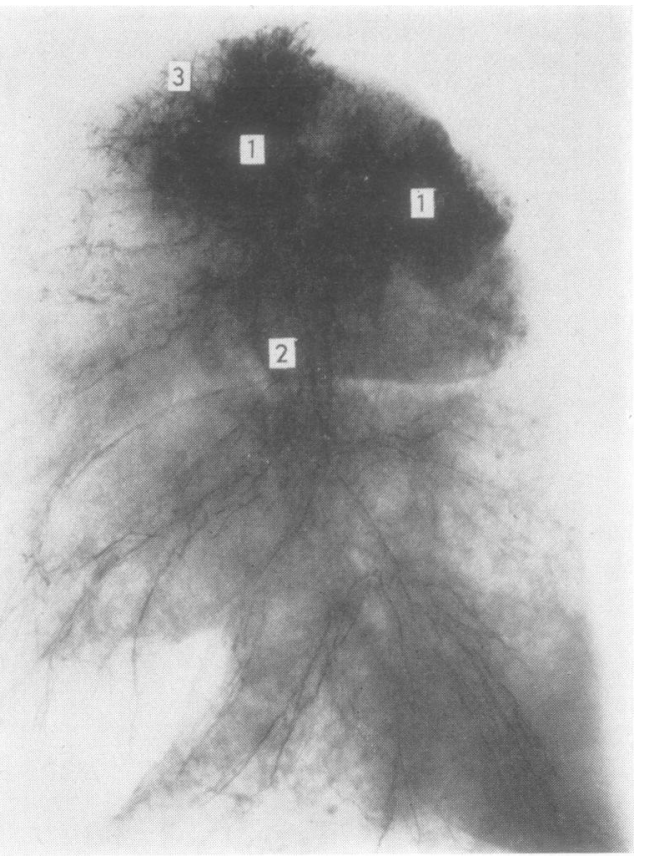

FIG. 3

FIG. 2.-Photomicrograph of lacunae in the tumour mass in Case 1. 1. Lacunae filled with injection mass; 2, extravasation of the injection material between tumour cells. $\times 13$.

FIG. 3.-Lateral radiograph of right lung of Case 3. 1, Tumour masses in apical and posterior segments of upper lobe; 2. proliferating bronchia! arteries: 3 , anastomotic filling of peripheral pulmonary arteries. (Right hilum faces $x$-ray tube.) $\frac{1}{4}$ of normal size.

Fig. 4.-Antero-posterior radiograph of left lung in Case 4. 1, Very large bronchial arteries; 2, tumour in apical segment of left upper lobe, showing extravasation of injection material. $\frac{1}{3}$ of normal size.

FIG. 5.-Lateral radiograph of right lung in Case 2. 1, Leash of dilated and new bronchial arteries leading to primary tumour: 2. primary tumour obstructing the right posterior basic bronchus; proliferating bronchial arteries. (Right hilum faces $x$-ray tube.) proliferating bro
1 of normal size.

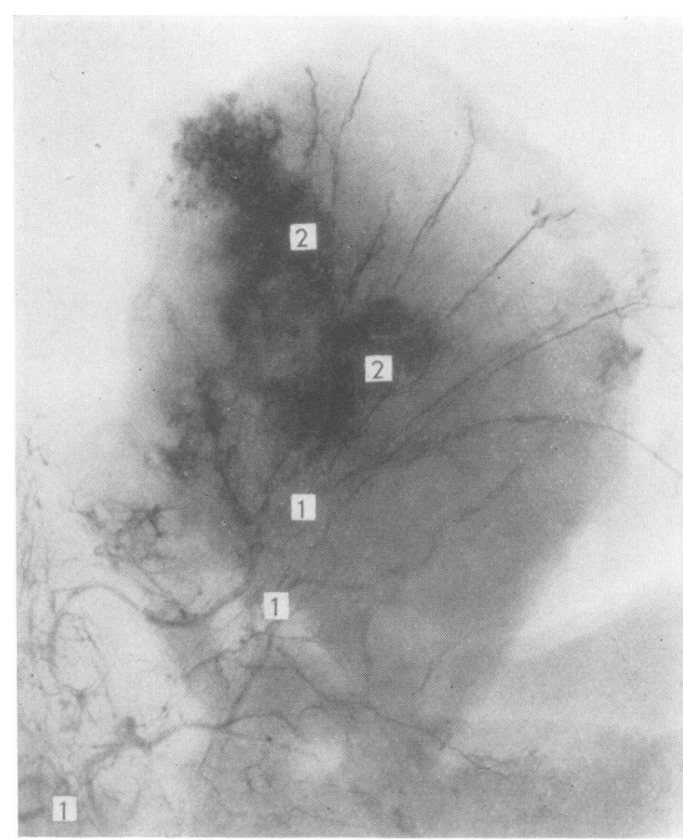

FIG 4

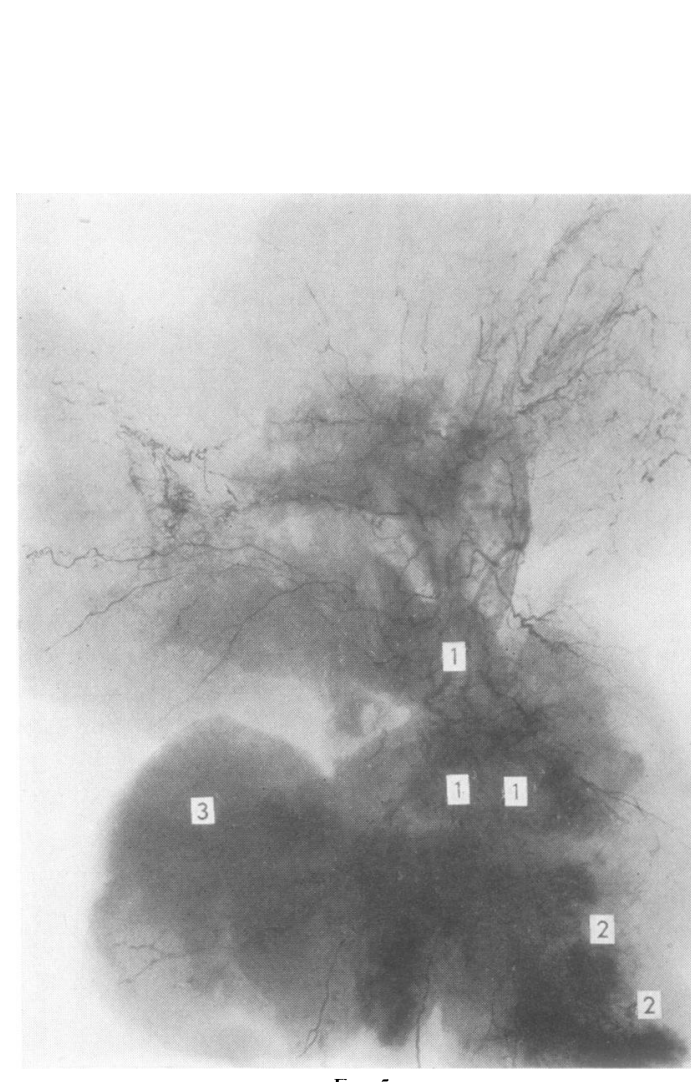

Fig. 5 


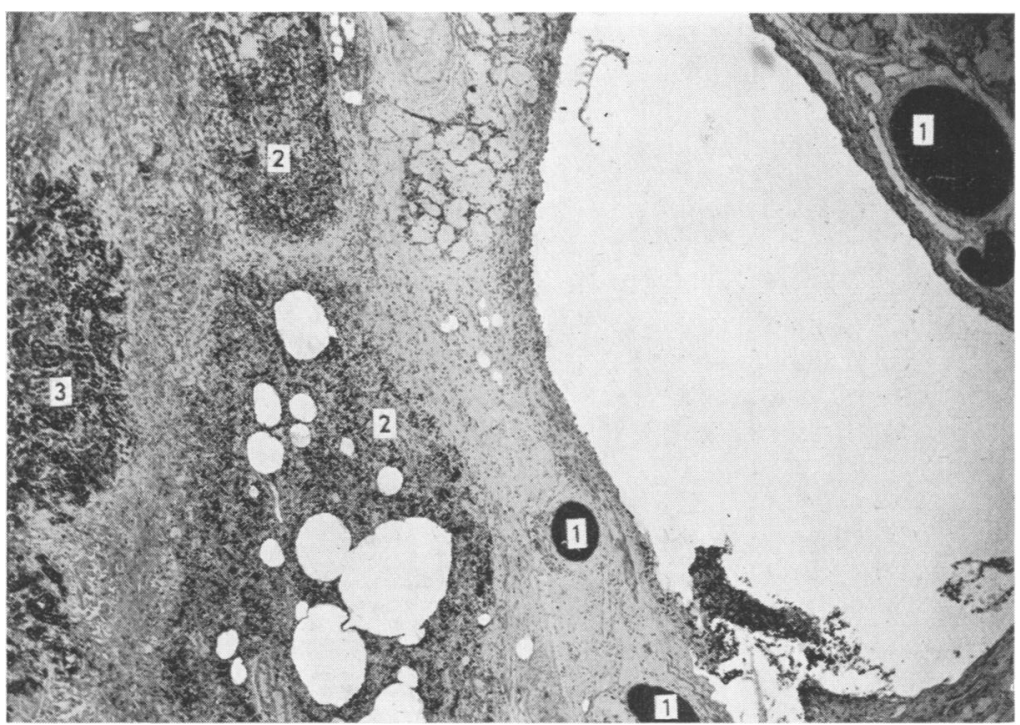

FIG.6.-Photomicrograph of right posterior basic bronchus in Case 2. 1, Grossly dilated bronchial arteries in the coat of bronchus; 2 , lumen is still patent, but tumour cells have invaded bronchial wall; 3 , tumour. $\times 28$.

A radiograph before death showed a shadow continuous with the right heart shadow and an additional shadow in the posterior basic segment of the right lower lobe. At necropsy $420 \mathrm{ml}$. of bloodstained fluid was present in the right pleural sac. A tumour was palpable near the right posterior basic bronchus and a similar mass was felt in the medial basic segment. There was massive right hilar lymph node enlargement. The pericardium was heavily infiltrated on the right side and the inferior vena cava was obstructed by a concentric pericardial mass $2 \mathrm{~cm}$. below its entry into the right atrium. A large thrombus obstructed its lumen.

Radiographic study showed filling of all bronchial arteries. The pattern to the left lung was normal. Distortion and proliferation of the bronchial artery pattern was seen in the right lower lobe, particularly in the right posterior basic segment proximal to the tumour. The medial basic segment showed the shadow of another mass, but the bronchial arteries in that area were normal in size and distribution (Fig. 5). No anastomotic filling of the pulmonary arteries had taken place.

Bronchography of the right lung showed obstruction of the right posterior basic bronchus with some stenosis of the apical lower lobe bronchus.

The histology of this case showed numerous large and well-injected bronchial arteries at the edge of the tumour and dilated injected lacunae within the tumour substance, which included the right posterior basic bronchus. The accompanying pulmonary artery was patent and free of the injection medium. The tumour cells were of the " oat cell " type (Fig. 6). Sections of the growth in the medial basic segment revealed an anaplastic mass which dis. placed the alveoli and bronchi in that area. In contrast to the tumour in the posterior basic segment no bismuthfilled vessels were seen in its substance or near its edge. The uninjected pulmonary arteries appeared to be dilated. The lymph nodes at the right hilum were heavily infiltrated with malignant cells, and large bronchial arterioles were seen in their substances and capsules.

Case 5.-An 85-year-old woman died from carcinoma of the stomach which had led to a fatal haemorrhage from an erosion of the lower thoracic

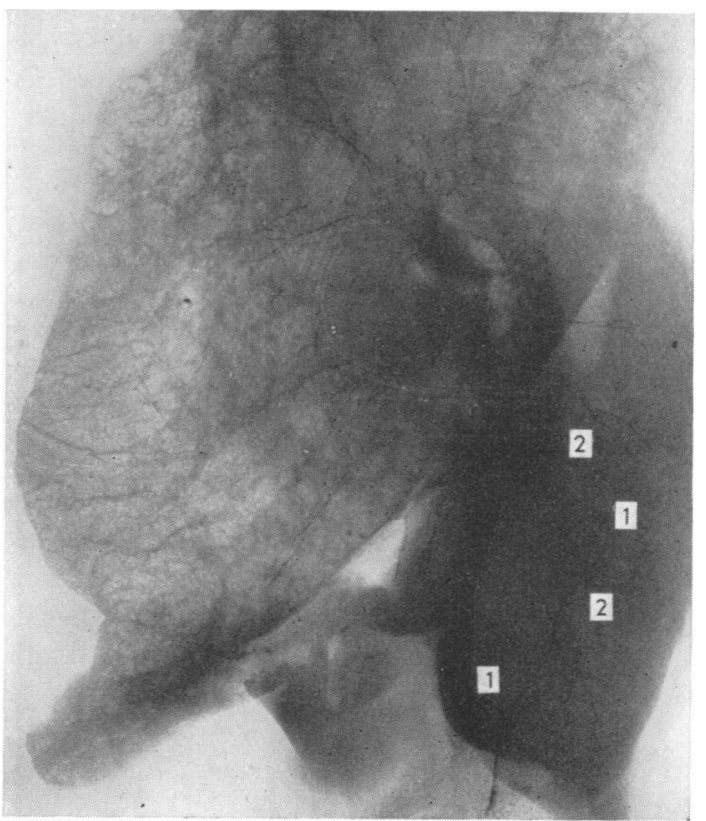

FIG. 7.-Lateral radiograph of right lung of Case 5. 1, Large tumour in right lower lobe; 2 , normal bronchial artery pattern in the right lower lobe. (Right hilum faces $x$-ray tube.) About $\frac{1}{4}$ of normal size. 
aorta. The liver was studded with many secondary deposits, and a very large mass was palpable in the lower lobe of the right lung.

The radiographic studies of the injected lungs showed a very dense mass in most of the right lower lobe, but the bronchial artery pattern throughout both lungs was normal (Fig. 7).

The histology of the mass in the right lower lobe showed a diffuse aggregate of anaplastic cells, suspended between collagenous fibrils. The walls of the bronchi appeared to be free from tumour cells, but the alveoli everywhere had massive infiltrations of malignant tissue. The pulmonary arteries in that lobe appeared to be very dilated, but were not injected. Small bronchial arteries were visible in the walls of the bronchi and no extension of these to the tumour was seen.

\section{Discussion}

The first four cases in this study indicate that bronchial carcinomata receive a profuse blood supply from the bronchial arteries. The pulmonary arteries accompanying the involved bronchi in Cases 1 and 3 were thrombosed, and recanalization of their lumina by injected vasa vasorum had taken place. Both of these patients showed clubbing of the fingers. The tumour in the medial basic segment of the right lower lobe of the second case showed absence of bronchial artery proliferation, and its cells resembled those seen in the secondary deposits in the hilar lymph nodes. It would therefore appear reasonable to regard this mass as a metastasis from the primary tumour in the posterior basic segment. The tumour in the right lower lobe of the fifth case was very probably a metastasis from the gastric carcinoma, and it is of interest that the bronchial arteries to that lobe remained unchanged. The present evidence supports the observations made by Wood and Miller (1938), who found proliferative changes in the bronchial arteries only in primary bronchial tumours. Their contention that haemoptyses come from the bronchial arteries may well be true in view of the large and thin-walled lacunae in the tumour substance which, in our cases, were seen to be in continuity with enlarged bronchial arteries. Presumably the pressure in these dilated vascular channels must be high and would exceed the pressure in similar blood spaces derived from the pulmonary arteries. The association of hypertrophic pulmonary osteoarthropathy with bronchial carcinoma is well recognized, and it is of interest that in two of our cases, where clubbing was prominent, thrombosis and recanalization of pulmonary artery branches by the vasa vasorum of the bronchial circulation could be demonstrated.

\section{SUMMARY}

The bronchial arteries in five cases of malignant pulmonary neoplasms were examined with the aid of a radio-opaque injection mass. In four cases of bronchial carcinoma a diffuse proliferative bronchial arterial pattern extending to the tumours was demonstrable. Large dilated lacunae derived from the bronchial arteries were seen in the tumours. In two cases of metastatic lung tumours no corresponding changes in the bronchial arteries were observed. Bronchopulmonary anastomoses were evident in two cases of primary bronchial neoplasm with hypertrophic pulmonary osteoarthropathy. The significance of these changes has been discussed.

We desire to express our thanks to Professor $\mathrm{J}$. McMichael and Professor J. H. Dible for the provision of facilities, to Dr. J. Crofton and to Dr. B. Lennox for their encouragement, to Miss M. S. McAdam for taking the many radiographs, to the technical staff of the Department of Pathology for preparing the slides for microscopy, to Mr. E. V. Willmott, F.R.P.S., for the photomicrography, and to Mr. A. K. Moreman, A.R.P.S., for printing the radicgraphs.

\section{REFERENCES}

Cudkowicz, L., and Armstrong, J. B. (1951). Thorax, 6, 343 Wood, D. A., and Miller, M. (1938). J. thorac. Surg., 7, 649 Wright, R. D. (1938). J. Path. Bact., 47, 489. 